\title{
IncRNA small nucleolar RNA host gene 12 promotes renal cell carcinoma progression by modulating the miR-200c-5p/collagen type XI $\alpha 1$ chain pathway
}

\author{
CONGJIE XU ${ }^{1 *}$, HUI LIANG ${ }^{2 *}$, JIAQUAN ZHOU $^{1}$, YANG WANG ${ }^{1}$, SHUAN LIU $^{1}$, \\ XIAOLIN WANG ${ }^{1}$, LIANGJU SU ${ }^{1}$ and XINLI KANG ${ }^{1}$ \\ Departments of ${ }^{1}$ Urology and ${ }^{2}$ Neurology, Hainan General Hospital, Haikou, Hainan 570105, P.R. China
}

Received May 24, 2019; Accepted June 23, 2020

DOI: $10.3892 / \mathrm{mmr} .2020 .11490$

\begin{abstract}
Renal cell carcinoma (RCC) is a primary malignant kidney cancer subtype. It has been suggested that long non-coding RNAs (lncRNAs) serve important roles in the progression of kidney cancer. In fact, the lncRNA small nucleolar RNA host gene 12 (SNHG12) was discovered to be overexpressed in various types of cancer. However, to the best of our knowledge, the role of SNHG12 in RCC remains unclear. The present study aimed to investigate the function of SNHG12 and its underlying molecular mechanism of action in RCC. In patient samples and datasets from The Cancer Genome Atlas. Reverse transcription-quantitative PCR, demonstrated that SNHG12 expression levels were upregulated in RCC tumor tissues, but not in normal kidney tissues. SNHG12 upregulation was also observed in RCC cell lines. Kaplan-Meier survival analysis indicated a poor prognosis for those patients with RCC who had upregulated SNHG12 expression levels. Following lentivirus transduction, SNHG12 was successfully knocked down (validated by western blot analysis) and cell migration and invasion assays were performed. SNHG12 knockdown markedly inhibited cell viability and invasion, while increasing apoptosis in both A498 and 7860 cell lines. The results of the luciferase reporter assay suggested that SNHG12 exerted its role by sponging microRNA (miR)-200c-5p, which led to the upregulation of its target gene, collagen type XI $\alpha 1$ chain (COL11A1). This was further validated, as miR-200c-5p inhibition reduced the effects of SNHG12 downregulation on cell viability and apoptosis, without affecting SNHG12 expression
\end{abstract}

Correspondence to: Dr Xinli Kang, Department of Urology, Hainan General Hospital, 19 Xiuhua Road, Xiuying, Haikou, Hainan 570105, P.R. China

E-mail: xinlikang2008@126.com

${ }^{*}$ Contributed equally

Key words: long non-coding RNA, small nucleolar RNA host gene 12 , renal cell carcinoma, microRNA-200c-5p, collagen type XI $\alpha 1$ chain levels. Furthermore, the findings indicated that SNHG12 may partially exert its role through COL11A1, which was also upregulated in RCC. In conclusion, the results of the present study suggested that the SNHG12/miR-200c-5p/COL11A1 axis may be crucial for RCC progression, which provided an insight into potential therapeutic strategies for RCC treatment.

\section{Introduction}

Kidney cancer is prevalent in both males and females, and renal cell carcinoma (RCC) is the most common form of kidney malignancy (1). Although treatment options for RCC have improved over the last decade from a non-specific immune approach (2), to therapies targeting vascular endothelial growth factor (VEGF) or tyrosine-protein kinase receptor UFO precursor (AXL) (3), the overall survival rate remains low. Although the five-year disease specific survival in patients with stage I-II reaches $80 \%$, it is only $60 \%$ in stage III and $<10 \%$ in stage IV patients (4). Therefore, understanding the molecular mechanisms of RCC development and progression is essential.

Long non-coding RNAs (lncRNAs) are non-protein coding transcripts of $>200$ nucleotides in length (5). Previous studies have suggested that lncRNAs have a prominent role in the competing endogenous RNA (ceRNA) network, in which they serve as microRNA (miRNA/miR) sponges, thus controlling the expression of genes associated with cancer development (6-8). For instance, the lncRNA HOX transcript antisense RNA (HOTAIR) was discovered to affect the proliferation and tumorigenesis of RCC by inhibiting the miR-217/hypoxia-inducible factor-1 (HIF-1) $\alpha / \mathrm{AXL}$ pathway (9). Additionally, lncRNA H19 was suggested to potentially mediate breast cancer cell plasticity by differentially sponging miR-200b/c and let-7b (10). Thus, these findings indicated that lncRNAs may be crucial regulators of cancer progression and metastasis (11-13). Nonetheless, their function in RCC remains poorly understood.

The expression levels of small nucleolar RNA host gene 12 (SNHG12), located on chromosome 1p35.3, were discovered to be upregulated in several types of cancer, including non-small cell lung cancer, glioma, cervical cancer and bladder cancer (14-17). In previous studies, SNHG12 was also reported 
to function as an miRNA sponge and influence several tumor-related pathways, such as the $\mathrm{Wnt} / \beta$-catenin signaling pathway and the Jak/STAT3 signaling pathway (15,17-19). In addition, SNHG12 was identified to serve as an oncogene, where it had a crucial role in tumor progression $(20,21)$. SNHG12 has also been associated with the unfavorable prognosis of nasopharyngeal carcinoma and gastric cancer, where it served as a potential biomarker for cancer progression $(22,23)$. However, to the best of our knowledge, the role of SNHG12 in RCC remains unknown.

In the present study, SNHG12 expression levels were investigated in samples obtained from patients with RCC. SNHG12 knockdown significantly inhibited the viability and invasion of RCC cell lines, while increasing apoptosis. Notably, SNHG12 was discovered to directly interact with miR-200c-5p, which indicated that it may serve as a sponge and control collagen type XI $\alpha 1$ chain (COL11A1) expression levels. In conclusion, the findings of the present study suggested that the SNHG12/miR-200c-5p/COL11A1 axis may be involved in RCC progression.

\section{Materials and methods}

Clinical samples. A total of 58 patients (36 males and 22 females; age, 31-72 years) diagnosed with RCC by pathological examination after surgery at The Department of Urology, Hainan General Hospital (Haikou, China) (August 2014-August 2017) were enrolled in the present study. Patients who received chemotherapy, radiotherapy or any other therapy were excluded from the present study. Tumor tissues and paired adjacent non-tumor renal tissues were collected and stored at $-80^{\circ} \mathrm{C}$ for subsequent RNA extraction. Patients were classified as RCC according to the World Health Organization criteria (24) and tumor staging was determined using the tumor-node-metastasis (TNM) classification (4). The study was approved by the Medical Ethics Committee of Hainan General Hospital (Haikou, China). Written, informed consent was obtained from all 58 patients.

TCGA database. RNA sequencing and clinical data of patients with RCC were downloaded from Genomic Data Commons Data Portal (portal.gdc.cancer.gov). Patient data were excluded if $>75 \%$ lncRNA data was missing or if survival information was not complete. Patients $>18$ years old were included in the study. After screening, a total of 278 patients with RCC and 33 non-tumor kidney samples were used in the present study (25). TCGA data was analyzed using RStudio version 0.99 .896 software (RStudio, Inc.). Survival analysis were conducted using Kaplan-Meier plots by SPSS version 22.0 software (IBM, Corp.).

Cell culture. Human renal cell carcinoma cell lines A498, 786O, Caki-1, Caki-2 and ACHN, and the normal renal epithelial cell line HK-2 as well as $293 \mathrm{~T}$ cell line were purchased from the American Type Culture Collection. Cells were cultured in RPMI-1640 medium, supplemented with $10 \%$ FBS and 1\% penicillin/streptomycin (all from Gibco; Thermo Fisher Scientific, Inc.). All cells were maintained in a humidified incubator with $5 \% \mathrm{CO}_{2}$ at $37^{\circ} \mathrm{C}$.
Cell transfection. Small interfering RNAs (siRNAs/si) targeting SNHG12 and negative control (NC; si-NC) were synthesized by Guangzhou RiboBio Co., Ltd. using the following sequences: Si-SNHG12 sense, 5'-GCAGUGUGC UACUGAACUUTT-3' and antisense, 5'-AAGUUCAGUAGC ACACUGCTT-3'; and si-NC sense, 5'-UUCUCCGAACGU GUCACGUTT-3' and antisense, 5'-ACGUGACACGUUCGG AGAATT-3'. Si-COL11A1 was purchased from Sigma-Aldrich; Merck KGaA. si-NC for si-COL11A1 was the same with si-SNHG12. The miR-200c mimics, miR-NC, miR-200c inhibitor, inhibitor-NC, pcDNA SNHG12 and pcDNA3.1 empty vector were purchased from GeneCopoeia, Inc. The sequences were as follows: Si-COL11A1 sense, 5'-UUCUAAAUUUGA UGGUUUGCGTT-3' and antisense, 5'-CAAACCAUCAAA UUUAGAAGA-3'; miR-200c-5p mimics, 5'-UAAUACUGC CGGGUAAUGAUGGA-3'; miR-mimics-NC, 5'-UCACAA CCUCCUAGAAAGAGUAGA-3'; and miR-200c-5p inhibitor, 5'-UAAUACUGCCGGGUAAUGAUGGA-3'; inhibitor-NC, 5'-CGAACUUAUCUUAGGUACUC-3'. Briefly, 1x106 A498 or 7860 cells were seeded into six-well plates and transfected with $50 \mathrm{nM}$ siRNAs, $30 \mathrm{nM}$ mimics, $30 \mathrm{nM}$ inhibitors, $2 \mu \mathrm{g}$ pcDNA SNHG12 or pcDNA 3.1 empty vector using Lipofectamine ${ }^{\circledR} 2000$ reagent (Invitrogen; Thermo Fisher Scientific, Inc.), according to the manufacturer's protocol. After $48 \mathrm{~h}$ of transfection at $37^{\circ} \mathrm{C}$, the transfection efficiency was determined by reverse transcription-quantitative PCR (RT-qPCR). Untransfected RCC cells were used as the 'Blank' control, and these cells were treated with nothing.

$R T-q P C R$. Total RNA was extracted from cells using TRIzol ${ }^{\circledR}$ reagent (Invitrogen; Thermo Fisher Scientific, Inc.) and reverse transcribed into cDNA using PrimeScript Reverse Transcriptase (Takara Biotechnology Co., Ltd.). RT was performed using the following procedure: $30^{\circ} \mathrm{C}$ for $10 \mathrm{~min}$ followed by $42^{\circ} \mathrm{C}$ for $45 \mathrm{~min}$. qPCR was subsequently performed using the SYBR Premix Taq kit (Takara Biotechnology Co., Ltd.) on a LightCycler Real-Time PCR instrument [Roche Diagnostics (Shanghai) Co., Ltd.]. qPCR was performed using the following conditions: Enzyme activation at $95^{\circ} \mathrm{C}$ for $2 \mathrm{~min}, 1$ cycle; 40 cycles of denaturation at $95^{\circ} \mathrm{C}$ for $10 \mathrm{sec}$ and annealing/extension $60^{\circ} \mathrm{C}$ for $60 \mathrm{sec}$. U6 was used as the endogenous control for miR-200c-5p and SNHG12, while GAPDH was used as the loading control for COL11A1. The following primer sequences were used for the qPCR: SNHG12 forward, 5'-AGGGCCATGTAACCA GTGAA-3' and reverse, 5'-GCTGGCCTTAATCTGACT GC-3'; U6 forward, 5'-GCTTCGGCAGCACATATACTA AAAT-3' and reverse, 5'-CGCTTCACGAATTTGCGTGTC AT-3'; miR-200c-5p forward, 5'-TACATCATAATACTGCCG GGTAA-3' and reverse, 5'-GGATTGGATGTTCTCCACAGT CTC-3'; COL11A1 forward, 5'-AGTGGCATCGGGTAGCAA TCA-3' and reverse, 5'-TGTCCCCCTCAAAAACTTCTT CAT-3'; and GAPDH forward, 5'-CGCTCTCTGCTCCTC CTGTTC-3' and reverse, 5'-ATCCGTTGACTCCGACCTTCA C-3'. The relative expression levels of each target were quantified using the $2^{-\Delta \Delta \mathrm{Cq}}$ method (26).

Western blotting. Cells were washed with PBS and total protein was extracted using RIPA lysis buffer (Invitrogen; Thermo Fisher Scientific, Inc.) containing a protease inhibitor cocktail 
(Roche Diagnostics Co., Ltd.). Total protein was quantified using a bicinchoninic acid protein assay kit (Pierce; Thermo Fisher Scientific, Inc.) and $40 \mu \mathrm{g}$ protein/well was separated via 10\% SDS-PAGE. The separated proteins were subsequently transferred onto PVDF membranes (EMD Millipore) and blocked with $5 \%$ non-fat milk at room temperature for $1 \mathrm{~h}$. The membranes were then incubated with the following primary antibodies overnight at $4^{\circ} \mathrm{C}$ : Anti-COL11A1 (cat. no. ab64883; Abcam) and anti- $\beta$-actin (cat. no. ab6276; Abcam). Antibodies were used at 1:1,000 dilution. Following the primary antibody incubation, the membranes were washed with TBS- $0.05 \%$ Tween-20 (TBST) 4-6 times for $1 \mathrm{~h}$, then incubated with a horseradish peroxidase-conjugated secondary antibody for $1 \mathrm{~h}$ at room temperature (Goat anti-rabbit and anti-mouse IgG, cat. nos. ab205718 and ab205719, respectively; both Abcam), secondary antibodies were used at 1:5,000 dilution. The membranes were subsequently washed with TBST 4-6 times for $1 \mathrm{~h}$ and protein bands were visualized using an ECL substrate kit (Abcam). ImageJ software (version 1.8.0; National Institutes of Health) was used for densitometry.

Transwell invasion assay. Transwell invasion assays were performed using $8-\mu \mathrm{m}$ pore Matrigel ${ }^{\circledR}$-precoated $\left(37^{\circ} \mathrm{C}\right.$ for $3 \mathrm{~h}$ and room temperature overnight) polycarbonate membranes (BD Biosciences). Briefly, $3 \times 10^{4}$ cells were seeded into the upper chambers of Transwell plates in serum-free DMEM (Gibco; Thermo Fisher Scientific, Inc.). The lower chambers contained complete DMEM (Gibco; Thermo Fisher Scientific, Inc.) supplemented with $10 \%$ FBS. Following incubation at $37^{\circ} \mathrm{C}$ for $48 \mathrm{~h}$, cells in the upper chamber were removed and the invasive cells on the lower surface of the membranes were fixed in $4 \%$ paraformaldehyde at room temperature for $15 \mathrm{~min}$, stained with $0.5 \%$ crystal violet at room temperature for $20 \mathrm{~min}$ and visualized using a light microscope (magnification, x100) (Olympus Corporation). ImageJ software (version 1.8.0; National Institutes of Health) was used for data analysis.

Flow cytometric analysis of apoptosis. Early and late (top and lower right corner of the flow dot plot, respectively) stage apoptosis were detected by flow cytometric analysis. Briefly, $0.5 \times 10^{6} \mathrm{~A} 498$ or 7860 cells were seeded to six-well plates and transfected with the transfectants for $48 \mathrm{~h}$ at $37^{\circ} \mathrm{C}$. The cells were subsequently harvested (Eppendorf centrifuge 5418R) and washed twice with PBS. The cells were then resuspended in binding buffer (cat. no. BDB556454; BD Biosciences), and stained with Annexin V-FITC and 7-Aminoactinomycin D (AAD) using an Annexin V-FITC/7-AAD Apoptosis Detection kit (BD Biosciences), according to the manufacturer's protocol. Apoptotic cells were subsequently analyzed using a BD FACSVerse flow cytometer (BD Biosciences) and analyzed using FlowJo version 10.0.7 software (FlowJo LLC).

MTT assay. A total of $2 \times 10^{4}$ cells were seeded/well into a 96-well plate and incubated for 24,48 or $72 \mathrm{~h}$ at $37^{\circ} \mathrm{C}$. Cell viability was subsequently analyzed in triplicate using an MTT assay [Roche Diagnostics (Shanghai) Co., Ltd.], according to the manufacturer's protocol. Then, $10 \mu 1$ MTT solution was added to each well and DMSO was used to dissolve purple formazan. Cell viability was analyzed at an absorbance
Table I. Association between SNHG12 expression levels and clinicopathological characteristics in patients with renal cell carcinoma $(\mathrm{n}=58)$.

\begin{tabular}{|c|c|c|c|}
\hline \multirow[b]{2}{*}{ Parameter } & \multicolumn{2}{|c|}{$\begin{array}{l}\text { SNHG12 } \\
\text { expression } \\
\text { levels }\end{array}$} & \multirow[b]{2}{*}{ P-value } \\
\hline & Low, $\mathrm{n}$ & High, $n$ & \\
\hline Age, years & & & 0.905 \\
\hline$>60$ & 7 & 15 & \\
\hline$\leq 60$ & 12 & 24 & \\
\hline Sex & & & 0.455 \\
\hline Male & 16 & 20 & \\
\hline Female & 12 & 10 & \\
\hline Histological differentiation & & & $<0.001$ \\
\hline High & 23 & 4 & \\
\hline Low & 9 & 22 & \\
\hline Lymph node metastasis & & & 0.018 \\
\hline $\mathrm{N} 0-\mathrm{N} 1$ & 20 & 9 & \\
\hline N2-NX & 11 & 18 & \\
\hline Distant metastasis & & & 0.018 \\
\hline M0 & 18 & 11 & \\
\hline M1-MX & 9 & 20 & \\
\hline Tumor node metastasis stage & & & 0.001 \\
\hline $\mathrm{T} 1-\mathrm{T} 2$ & 19 & 10 & \\
\hline T3-T4 & 6 & 23 & \\
\hline Tumor size & & & 0.001 \\
\hline$\leq 4 \mathrm{~cm}$ & 20 & 9 & \\
\hline$>4 \mathrm{~cm}$ & 5 & 24 & \\
\hline
\end{tabular}

SNHG12, small nucleolar RNA host gene 12 .

at $570 \mathrm{~nm}$ using an iMark microplate absorbance reader (Bio-Rad Laboratories, Inc.).

Luciferase reporter assay. TargetScan (targetscan. org/mamm_31) and miRcode (mircode.org) were used for the prediction of miRNA targets. The putative miR-200c-5p binding site of the SNHG12 and COL11A1 3'-untranslated region (UTR) sequences were amplified and cloned into the pmirGLO vector (Promega Corporation) to synthesize SNHG12-WT and COL11A1-WT, respectively. Mutant (MUT) 3'-UTR fragments were generated by site-directed mutagenesis (InFusion HD Cloning Plus; Takara Biotechnology Co., Ltd.) and then were cloned into the pmirGLO plasmid (referred to as SNHG12-MUT and COL11A1-MUT, respectively). Then, $2 \times 10^{5} 293 \mathrm{~T}$ cells were seeded into 24 -well plates and cultured for $24 \mathrm{~h}$ at $37^{\circ} \mathrm{C}$. The cells were then co-transfected with $5 \mu \mathrm{g}$ SNHG12- or COL11A1-WT/MUT luciferase constructs, and miR-200c-5p mimics (Lipofectamine 2000; Thermo Fisher Scientific, Inc.). Following transfection for $48 \mathrm{~h}$ at $37^{\circ} \mathrm{C}$, cells were collected and luciferase activity was detected using a Dual-Luciferase Reporter Assay system (Promega Corporation). Renilla Luciferase was used for normalization. 

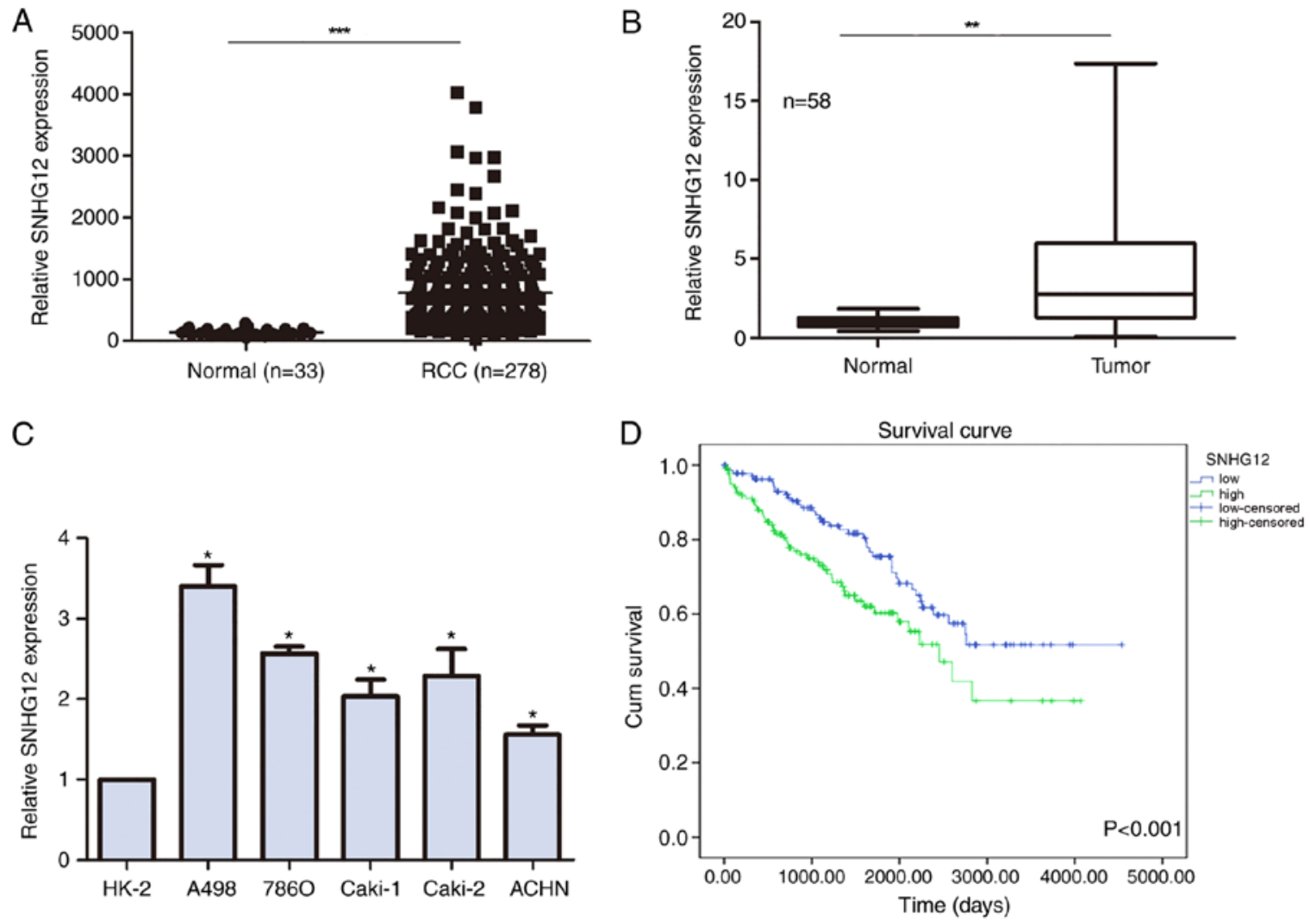

Figure 1. Relative expression levels of SNHG12 are upregulated in patients with RCC and in RCC cell lines. (A) SNHG12 expression levels in patients with RCC and normal patient samples from TCGA database. (B) SNHG12 expression levels in clinical RCC samples compared with adjacent non-tumor tissues were analyzed using RT-qPCR. Data are expressed in the form of taking logarithm of the counts number to the base 2. (C) SNHG12 expression levels in RCC cell lines and the HK-2 normal kidney cell line were analyzed using RT-qPCR. (D) Kaplan-Meier curve of the survival of patients with RCC from TCGA database according to low and high SNHG12 expression levels. 0, alive; 1, deceased. SNHG12, small nucleolar RNA host gene 12; RCC, renal cell carcinoma; TCGA, The Cancer Genome Atlas; RT-qPCR, reverse transcription-quantitative PCR; Cum, cumulative. ${ }^{*} \mathrm{P}<0.05,{ }^{* * *} \mathrm{P}<0.01,{ }^{* * * *} \mathrm{P}<0.001$.

Statistical analysis. Statistical analysis was performed using SPSS Statistics 22.0 software (IBM Corp.). Pairwise comparisons were assessed using an unpaired Student's t-test or a paired Student's t-test when the data were paired (Fig. 1B). Multigroup comparisons were performed using two-way ANOVA, followed by a Bonferroni's post hoc test. A Kaplan-Meier curve was used for survival analysis and the P-value was obtained using the log-rank test. A $\chi^{2}$ test was used to determine the association between SNHG12 expression levels and clinicopathological variables, whereas a Spearman's correlation analysis test was used to determine the correlation between SNHG12 and miR-200c-5p expression levels. All experiments were conducted in triplicate. Data are presented as the mean \pm SD. $P<0.05$ was considered to indicate a statistically significant difference.

\section{Results}

SNHG12 expression levels are upregulated in RCC patient samples and RCC cell lines. The expression levels of SNHG12 were compared between RCC and non-tumor samples from TCGA database; SNHG12 expression levels were discovered to be significantly upregulated in patients with RCC compared with in non-tumor patient samples $(799.9 \pm 35.41$ vs. $141 \pm 8.625$ normalized counts, respectively; Fig. 1A). To validate this finding, SNHG12 expression levels were also analyzed in clinical samples and similarly, SNHG12 expression levels were significantly upregulated in the RCC tumor tissues compared with the adjacent non-tumor tissues (Fig. 1B). The expression levels of SNHG12 were also evaluated in RCC cell lines, all of which demonstrated upregulated expression levels of SNHG12 compared with the normal kidney epithelial cell line HK-2 (Fig. 1C).

Association between SNHG12 expression levels and clinical characteristics in patients with RCC. The clinical characteristics analyzed in the present study included age, sex, histological differentiation, TNM stage, tumor size and lymph node and distant metastases (Table I). The median expression levels of SNHG12 were used to categorize patients into 'high' and 'low' expression groups. High SNHG12 expression levels were significantly associated with a lower histological differentiation advanced TNM stage and further lymph node and distant metastases. TCGA datasets were then used to further investigate whether SNHG12 expression levels influenced the survival of patients with RCC; high SNHG12 expression levels in patients with RCC were associated with a shorter overall survival rate compared with low expression levels (Fig. 1D).

SNHG12 promotes cell viability and invasion and inhibits apoptosis in RCC cell lines. To determine the function of SNHG12 in RCC cells, siRNA was used to knockdown 
A

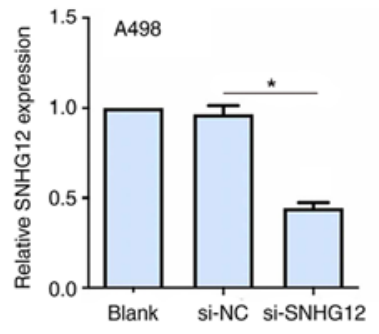

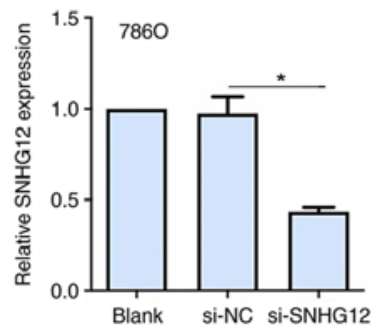

B
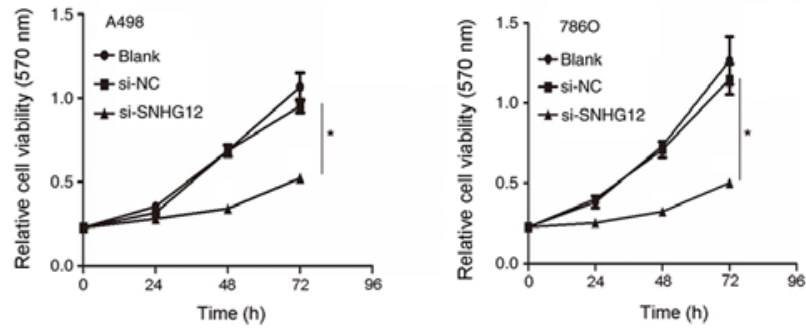

C
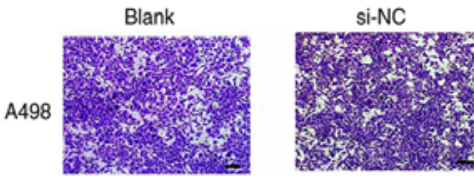

si-SNHG12

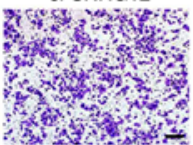

7860
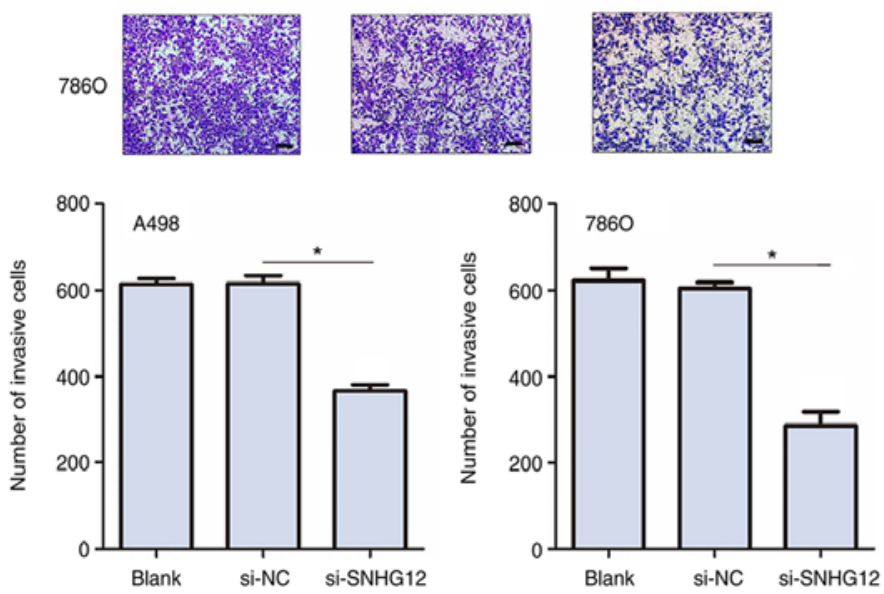

D
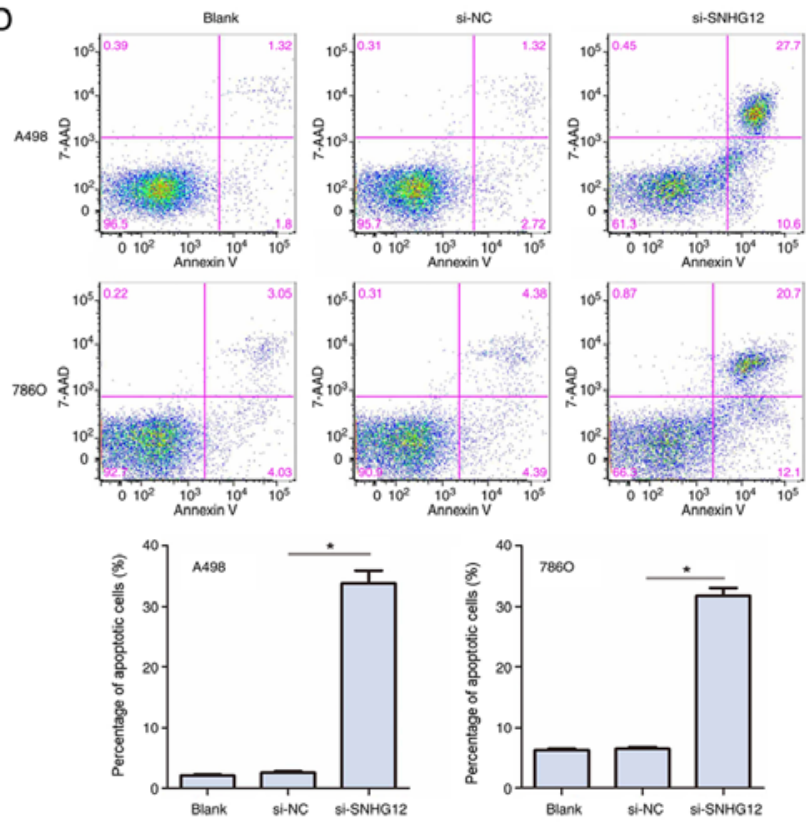

Figure 2. Functional role of SNHG12 in RCC cell lines. (A) Transfection efficiency of si-SNHG12 in A498 and 7860 cell lines was analyzed using reverse transcription-quantitative PCR. (B) Cell viability was analyzed using an MTT assay following SNHG12 knockdown. (C) Cell invasive ability was detected using a Transwell assay following SNHG12 knockdown. Magnification, x100; scale bar, $100 \mu \mathrm{m}$. (D) Flow cytometric analysis of apoptosis was performed following SNHG12 knockdown. Untransfected RCC cells were used as the 'blank' control. *P<0.05. SNHG12, small nucleolar RNA host gene 12; RCC, renal cell carcinoma; si, small interfering RNA; NC, negative control; 7-AAD, 7-Aminoactinomycin D.

SNHG12 expression levels in the A498 and 7860 cell lines. In both cell lines, the transfection with si-SNHG12 significantly downregulated SNHG12 expression levels compared with the si-NC groups (Fig. 2A). An MTT assay was subsequently performed to determine the cell viability in the transfected RCC cells. SNHG12 knockdown significantly reduced cell viability at 48 and $72 \mathrm{~h}$ post transfection compared with the si-NC group in both cell lines (Fig. 2B). To investigate the invasive properties of the transfected RCC cells, a Transwell invasion assay was used. The knockdown of SNHG12 expression levels significantly inhibited the invasive ability of A498 and 7860 cells $(\mathrm{P}<0.05$; Fig. 2C). Flow cytometric analysis also demonstrated that the knockdown of SNHG12 expression levels significantly increased the percentage of apoptotic cells in both cell lines compared with the si-NC group (Fig. 2D). Altogether, these results indicated that SNHG12 may promote cell viability and invasion, whilst inhibiting cell apoptosis in RCC cell lines.

SNHG12 downregulates the expression levels of miR-200c-5p. To further investigate the mechanism of SNHG12 in the development of RCC, miRcode was used to screen for miRNAs predicted to bind to SNHG12, and SNHG12 was discovered to contain putative binding sites for miR-200c-5p. To validate this result, a correlation analysis was conducted between SNHG12 and miR-200c-5p expression levels in clinical RCC patient samples $(n=58)$. The expression levels of miR-200c-5p were revealed to be inversely correlated with SNHG12 expression levels ( $\mathrm{r}=-0.52 ; \mathrm{P}=0.032 ;$ Fig. $3 \mathrm{~A})$.

Thus, todetermine theinteraction between miR-200c-5p and SNHG12, RCC cell lines were transfected with miR-200c-5p mimics and si-SNHG12; the transfection efficiency of the miR-200c-5p mimics was successful, as the expression levels were significantly upregulated in the miR-200c-5p mimics group (Fig. 3B). Subsequently, SNHG12 knockdown markedly upregulated miR-200c-5p expression levels, as indicated by RT-qPCR. Notably, no significant differences were observed in the SNHG12 expression levels following the overexpression of miR-200c-5p compared with the two control groups (Fig. 3C). Subsequently, a luciferase reporter assay revealed that the relative luciferase activity was decreased in 293T cells co-transfected with pmirGLO-SNHG12-WT and miR-200c-5p mimics compared with pmirGLO-SNHG12-MUT and miR-200c-5p mimics co-transfected cells ( $\mathrm{P}<0.05$; Fig. 3D), which further supported an interaction between SNHG12 and miR-200c-5p. 

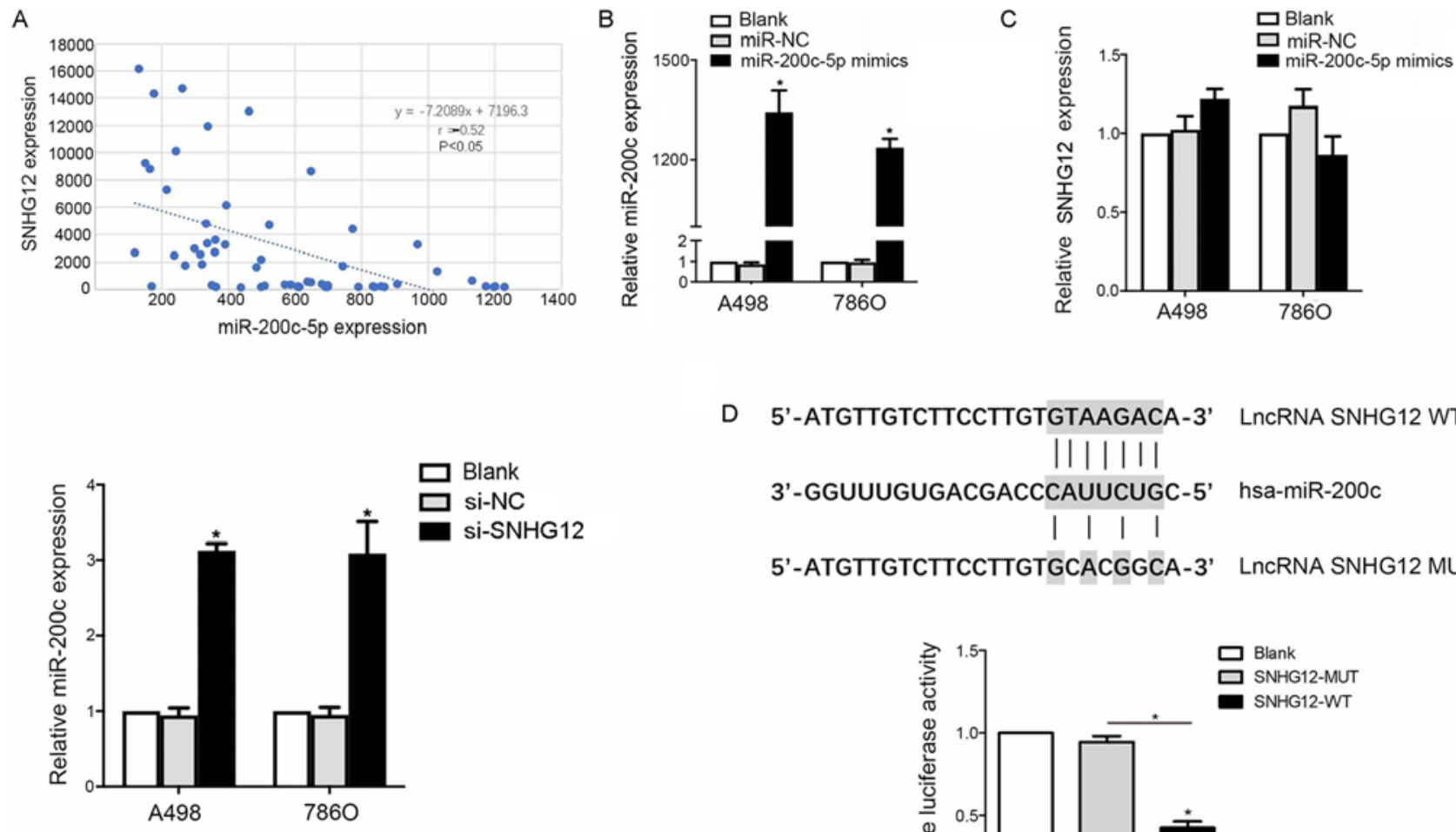

D 5'-ATGTTGTCTTCCTTGTGTAAGACA-3'
|||||| $\mid$
3'-GGCRNA SNHG12 WT
| | | |
5'-ATGTTGTCTTCCTTGTGCACGGCA-3' LncRNA SNHG12 MUT

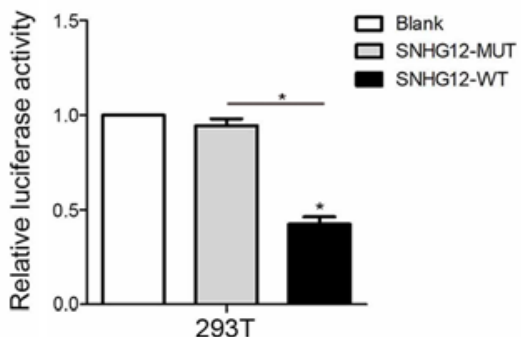

Figure 3. SNHG12 binds to miR-200c-5p and downregulates its expression levels. (A) Inverse correlation was identified between the expression levels of SNHG12 and miR-200c-5p in patient samples. (B) Transfection efficiency of miR-200c-5p mimics in A498 and 7860 cell lines was analyzed using reverse transcription-quantitative PCR. (C) Upregulation of miR-200c-5p expression levels in RCC cell lines had no effect on the expression levels of SNHG12. However, si-SNHG12 knockdown significantly upregulated miR-200c-5p expression levels. (D) Luciferase reporter assay demonstrated that the overexpression of miR-200c-5p reduced the intensity of the relative luciferase activity in 293T cells transfected with the SNHG12-WT vector. Untransfected RCC cells were used as the 'blank' control. "P $<0.05$. SNHG12, small nucleolar RNA host gene 12; RCC, renal cell carcinoma; miR, microRNA; NC, negative control; lncRNA, long non-coding RNA; WT, wild-type; MUT, mutant.

A

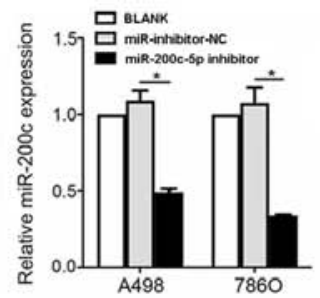

$\mathbf{C}$
$\vdots$
$\vdots$

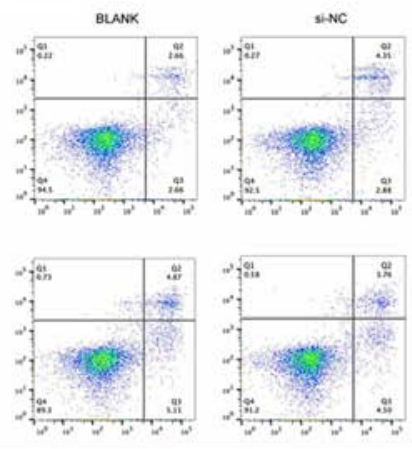

B
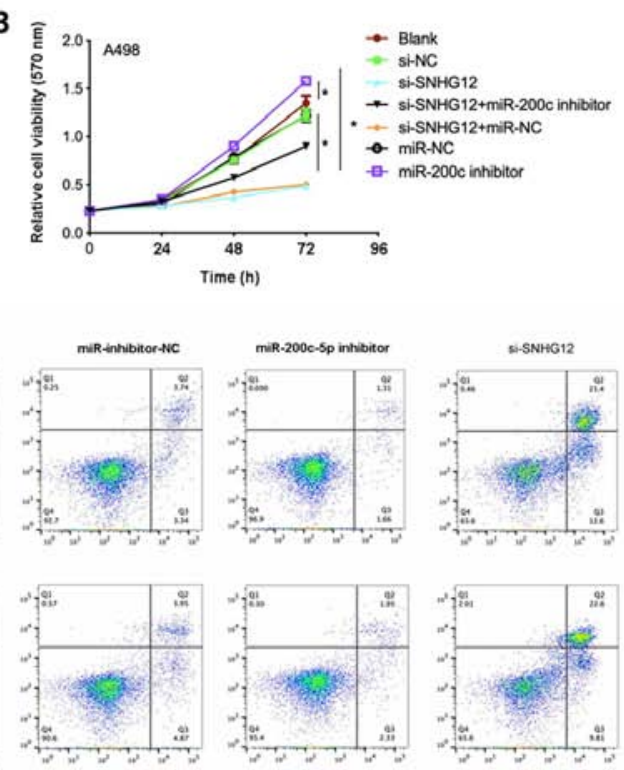

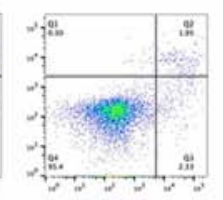

Annexin $V$
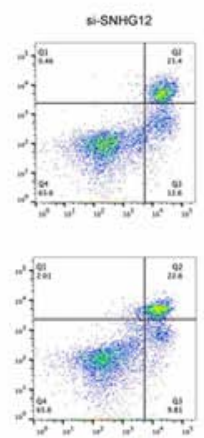
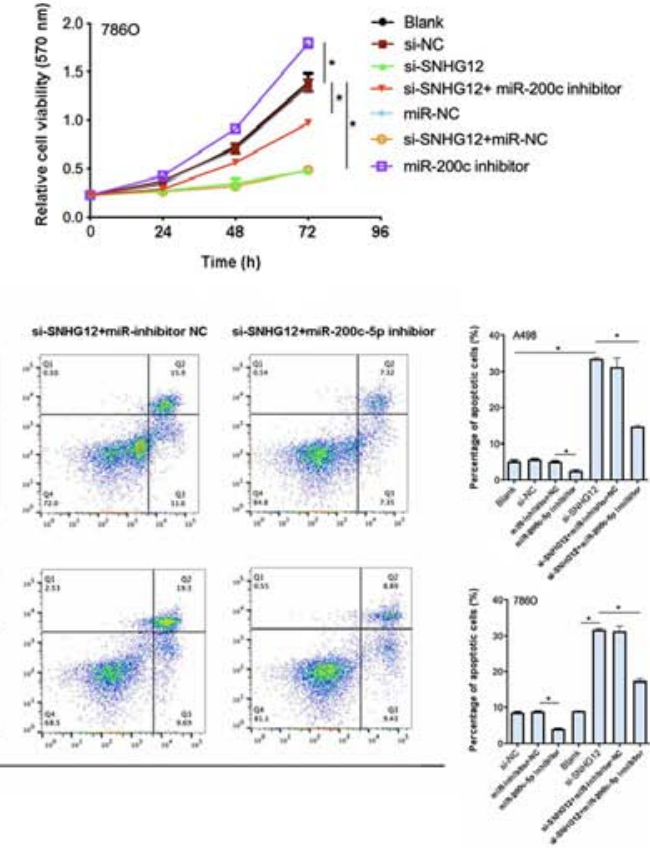

Figure 4. SNHG12 exerts its role by regulating miR-200c-5p. (A) Transfection efficiency of miR-200c-5p inhibitor in A498 and 7860 cell lines was determined using reverse transcription-quantitative PCR. (B) MTT assay was used to determine cell viability in RCC cells transfected with si-SNHG12 + miR-200c-5p inhibitor compared with cells transfected with si-SNHG12 alone. (C) Flow cytometric analysis of apoptosis following si-SNHG12 and miR-200c-5p inhibitor co-transfection compared with cells transfected with si-SNHG12 alone. Untransfected RCC cells were used as the 'blank' control. "P<0.05. SNHG12, small nucleolar RNA host gene 12; RCC, renal cell carcinoma; miR, microRNA; NC, negative control; si, small interfering RNA; 7-AAD, 7-Aminoactinomycin D. 
A
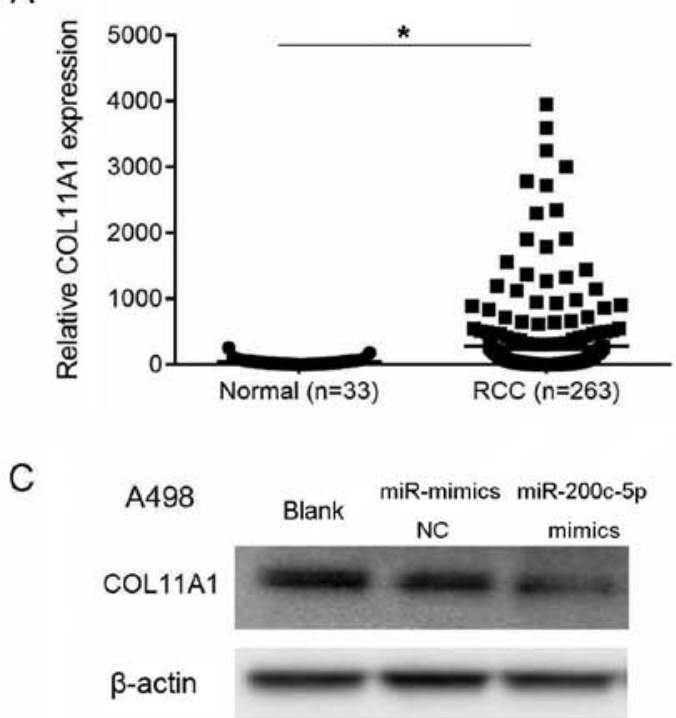

$7860 \quad$ Blank $\underset{\text { NC }}{\text { miR-mimics miR-200c-5p }}$

COL11A1

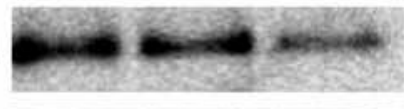

$\beta$-actin

D

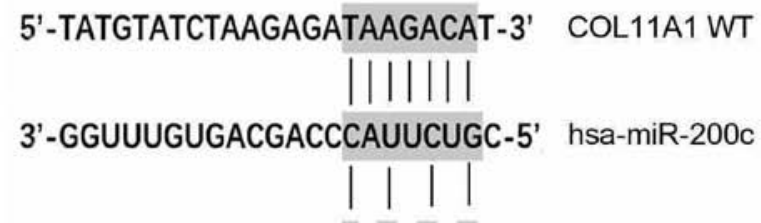

5'-TATGTATCTAAGAGATCAAAGAT-3' COL11A1 MUT
B
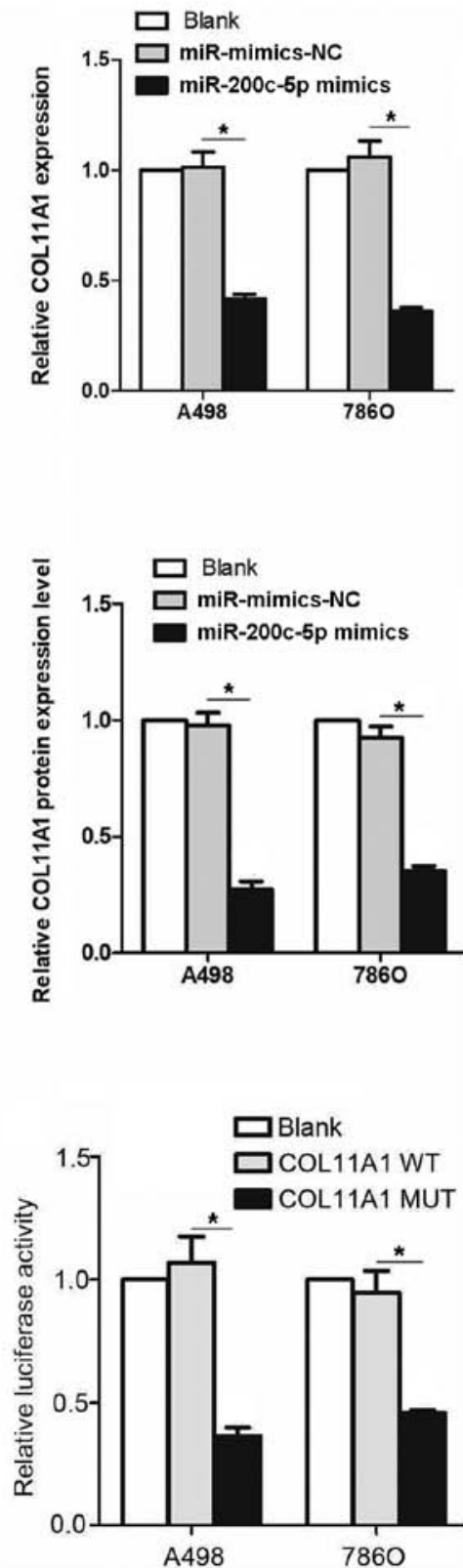

Figure 5. COL11A1 is a direct target of miR-200c-5p. (A) Relative COL11A1 expression levels in RCC tissues from The Cancer Genome Atlas database. $(\mathrm{P}=0.0248)(\mathrm{B})$ Relative COL11A1 expression levels following the transfection with the miR-200c-5p mimics in RCC cell lines was detected using reverse transcription-quantitative PCR. (C) Western blotting was used to determine the protein expression levels of COL11A1 in the blank, miR-mimics-NC and miR-200c-5p mimics group. (D) Luciferase reporter assay demonstrated a strong binding between the 3'-UTR of COL11A1 and miR-200c-5p. Untransfected RCC cells were used as the 'blank' control. "P<0.05. SNHG12, small nucleolar RNA host gene 12; COL11A1, collagen type XI $\alpha 1$ chain; RCC, renal cell carcinoma; miR, microRNA; NC, negative control; WT, wild-type; MUT, mutant; UTR, untranslated region. The binding sites of COL11A1 and miR-200c-5p were discovered using miRanda database.

SNHG12 exerts an oncogenic role through the regulation of $m i R-200 c-5 p$. To determine whether SNGH12 exerted its effects through miR-200c-5p, A498 and 7860 cell lines were co-transfected with si-SNHG12 and a miR-200c-5p inhibitor. The transfection of the miR-200c-5p inhibitor into both cell lines was successful, as the expression levels were significantly downregulated compared with inhibitor NC (Fig. 4A). The cell viability was significantly increased in both cell lines co-transfected with si-SNHG12 and the miR-200c-5p inhibitor compared with si-SNHG12 alone (Fig. 4B). In addition, in both cell lines, the percentage of apoptotic cells in the si-SNHG12 + miR-200c-5p inhibitor group was significantly decreased compared with the si-SNHG12 group $(\mathrm{P}<0.05$; Fig. 4C). Altogether, these observations suggested that SNHG12 may exert its oncogenic role through downregulating miR-200c-5p.

COL11A1 is a direct target of miR-200c-5p. TargetScan and miRcode were used to determine target genes of miR-200c-5p, which identified COL11A1 as one of the predicted targets. COL11A1 is associated with adhesion and extracellular matrix remodeling (27), which are both important processes in RCC.

The relative expression levels of COL11A1 were evaluated in datasets from patients with RCC and normal kidney 

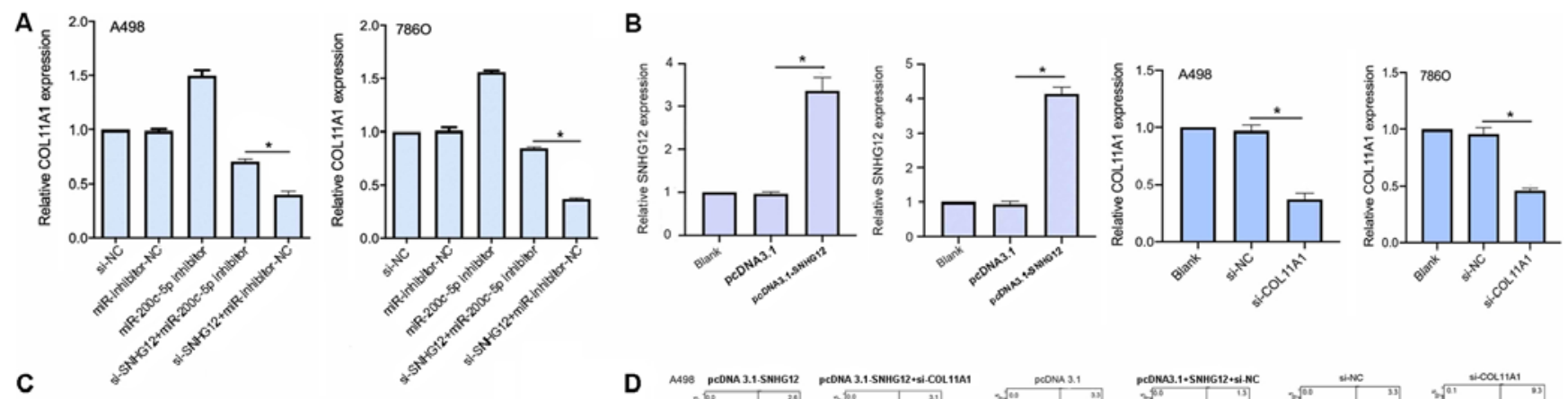

D
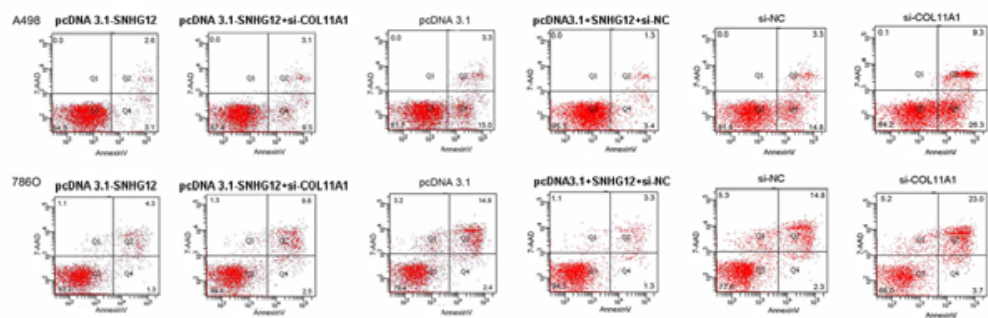

E
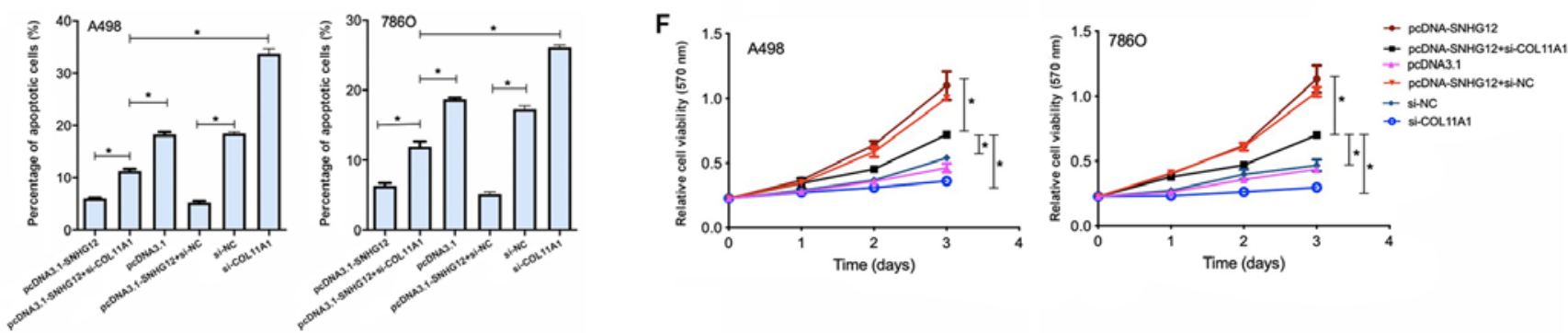

Figure 6. COL11A1 participates in the progression of RCC through the SNHG12/miR-200c-5p/COL11A1 axis. (A) COL11A1 expression levels were discovered to be regulated by SNHG12 in a miR-200c-5p-dependent manner. COL11A1 expression levels were analyzed using RT-qPCR. (B) Expression levels of SNHG12 and COL11A1 were analyzed using RT-qPCR following the transfection with pcDNA3.1-SNHG12 or si-COL11A1, respectively. (C) miR-200c-5p inhibitor rescued COL11A1 expression levels downregulated by si-SNHG12. (D) Flow cytometric analysis of apoptosis was performed in cells co-transfected with pcDNA3.1-SNHG12 and si-COL11A1. (E) Quantification of the apoptosis results were shown as bar plots. (F) Cell viability was determined using an MTT assay following the transfection with pcDNA3.1-SNHG12 + si-COL11A1. ${ }^{*} \mathrm{P}<0.05$. SNHG12, small nucleolar RNA host gene 12 ; COL11A1, collagen type XI $\alpha 1$ chain; miR, microRNA; NC, negative control; si, small interfering RNA; RT-qPCR, reverse transcription-quantitative PCR; 7-AAD, 7-Aminoactinomycin D.

tissues obtained from TCGA database. The expression levels of COL11A1 were significantly upregulated in the tissues from patients with RCC compared with the normal tissues (Fig. 5A). Subsequently, to determine whether COL11A1 was affected by miR-200c-5p, A498 and 7860 cell lines were transfected with miR-200c-5p mimics. The mRNA expression levels of COL11A1 were significantly downregulated following the transfection with the miR-200c-5p mimics (Fig. 5B). A similar trend was observed in the protein expression levels of COL11A1 (Fig. 5C). Moreover, the relative luciferase activity was significantly decreased in cells transfected with the COL11A1-WT 3'-UTR construct compared with the COL11A1-MUT 3'-UTR construct, which indicated that COL11A1 was a direct target of miR-200c-5p (Fig. 5D). The binding sites of COL11A1 and miR-200c-5p was discovered using miRanda database.

SNHG12 regulates COL11A1 expression levels through $m i R-200 c-5 p$. To investigate whether SNHG12 regulated COL11A1 expression levels by interacting with miR-200c-5p, COL11A1 mRNA expression levels were analyzed following the transfection with si-SNHG12 and the miR-200c-5p inhibitor. The transfection with si-SNHG12 significantly downregulated the expression levels of COL11A1 compared with the si-SNHG12 + miR-200c-5p inhibitor transfection
(Fig. 6A). Thus, these findings indicated that the inhibition of miR-200c-5p may partially reverse COL11A1 downregulation induced by si-SNHG12, indicating that SNHG12 may regulate COL11A1 expression levels through miR-200c-5p.

To confirm whether SNHG12 exerted its role through COL11A1, A498 and 7860 cell lines were transfected with the pcDNA3.1-SNHG12 overexpression vector or si-COL11A1. The transfection efficiency was proven to be successful (Fig. 6B and C), and MTT and apoptotic assays were subsequently performed. The cell viability was significantly decreased and the apoptotic rate was significantly increased in cells co-transfected with both pcDNA3.1-SNHG12 and si-COL11A1 compared with the cells transfected with pcDNA3.1-SNHG12 alone (Fig. 6D and E). These findings indicated that the effect of SNHG12 on the growth of RCC cell lines may be mediated, at least in part, by COL11A1.

\section{Discussion}

Previous studies have indicated that lncRNAs were vital to physiological and pathological processes (28-30). In fact, several lncRNAs have been associated with RCC; for example, in one study, the lncRNA metastasis associated lung adenocarcinoma transcript 1 interacted with miR-205 to promote 
aggressive RCC (31), whereas in another study, the lncRNA HOTAIR regulated HIF- $\alpha / A X L$ signaling by inhibiting miR-217 (9). Nevertheless, to the best of our knowledge, the role of SNHG12 in RCC remains unclear.

SNHG12 has been extensively investigated in several types of cancer, including papillary thyroid carcinoma, cervical cancer, lung cancer, osteosarcoma and hepatocellular carcinoma $(15,16,18,32,33)$. A previous study has reported that SNHG12 promoted tumor progression in non-small cell lung carcinoma by binding to miR-218 and miR-181a, which regulated Slug/zinc finger E-box binding homeobox (ZEB) 2 and mitogen activated protein kinase/Slug signaling (34).

The present study demonstrated that SNHG12 expression levels were upregulated in the tissues from patients with RCC compared with the normal kidney tissues. This finding was further validated in the A498 and 7860 RCC cell lines and patient samples. In addition, it was observed that SNHG12 knockdown significantly suppressed cell viability and invasion and promoted apoptosis in vitro. Moreover, analysis of TCGA data indicated that high SNHG12 expression levels were associated with poor clinical outcomes, a lower histological differentiation, advanced clinical stages and metastasis. Overall, these results suggested that SNHG12 may serve as an oncogene in RCC.

The ceRNA theory is widely accepted in the study of lncRNAs. According to the ceRNA theory, lncRNAs regulate gene expression by functioning as miRNA sponges, thereby interfering in the binding between miRNAs and target genes (35). Several studies have suggested that miR-200c-5p may function as a tumor suppressor gene in RCC (36-40). Indeed, miR-200c-5p inhibited RCC cell migration and invasion and participated in demethylation drug-induced decreased cell migration and invasion (41). Moreover, miR-200c-5p was reported to serve as a sponge for the lncRNA HOTAIR in the ceRNA network, whereby it regulated RCC progression through several oncogenes, including VEGFA, ZEB1 and ZEB2 (40). In the present study, miR-200c-5p was also discovered to interact with SNHG12, as validated by luciferase reporter assay, thus the findings suggested that SNHG12 may function as an oncogene in RCC through regulating COL11A1 expression levels by sponging miR-200c-5p. This was validated using a luciferase reporter assay, which demonstrated that the 3'-UTR of both SNH12 and COL11A1 were strongly bound to miR-200c-5p.

COL11A1, a primary interstitial extracellular matrix constituent, is one of the most frequently upregulated genes identified in chemoresistant tumors relative to normal tissues (42). Notably, COL11A1 was previously discovered to be associated with poor survival in patients with RCC, owing to its high expression levels in the tumor tissues compared with the normal kidney tissue (27). COL11A1 also promoted tumor progression in ovarian cancer (43) and it was associated with poor survival in multiple other types of cancer (e.g. pancreatic and colon cancer) (44-46). In addition, a previous study suggested that COL11A1 may be a highly specific biomarker and molecular signature of cancer-associated fibroblasts, as it was detected in several epithelial cancers, including lung cancer, ovarian cancer, head and neck cancer, brain cancer, glioma, colon cancer, liver cancer, bladder cancer, adrenocortical cancer, kidney cancer and mesothelioma, according to data from TCGA database (44). Thus, targeting COL11A1 or
COL11A1-associated genes may serve as an attractive therapeutic target for RCC.

The function of miR-200c-5p and COL11A1 was further analyzed in RCC cells using rescue assays, which indicated that miR-200c-5p upregulation increased the rate of cell apoptosis and decreased cell viability. COL11A1 knockdown also led to the same results, which was consistent with previous findings (27).

In conclusion, the findings of the present study suggested that SNHG12 may contribute to RCC progression by serving as an oncogene, sponging miR-200c-5p and thereby upregulating the expression levels of COL11A1. Thus, the present study provided further insight into the role of SNHG12 in RCC development.

\section{Acknowledgements}

Not applicable.

\section{Funding}

No funding was received.

\section{Availability of data and materials}

The datasets analyzed during the current study are available in the TCGA database (portal.gdc.cancer.gov/).

\section{Authors' contributions}

CX, YW, SL and XW conceived and designed the study, performed experiments, and drafted and revised the manuscript. HL and LS performed experiments and collected data.JZ performed experiments. XK funded the study and interpreted the data. All authors read and approved the final manuscript.

\section{Ethics approval and consent to participate}

The present study was approved by the Medical Ethics Committee of Hainan General Hospital (Haikou, China). Written informed consent was obtained from all patients.

\section{Patient consent for publication}

Not applicable.

\section{Competing interests}

The authors declare that they have no competing interests.

\section{References}

1. Barata PC and Rini BI: Treatment of renal cell carcinoma: Current status and future directions. CA Cancer J Clin 67: 507-524, 2017.

2. Choueiri TK and Motzer RJ: Systemic therapy for metastatic renal-cell carcinoma. N Engl J Med 376: 354-366, 2017.

3. Garcia JA and Rini BI: Recent progress in the management of advanced renal cell carcinoma. CA Cancer J Clin 57: 112-125, 2007.

4. Jonasch E, Gao J and Rathmell WK: Renal cell carcinoma. BMJ 349: g4797, 2014. 
5. Chi Y, Wang D, Wang J, Yu W and Yang J: Long non-coding RNA in the pathogenesis of cancers. Cells 8: 1015, 2019.

6. Schmitt AM and Chang HY: Long noncoding RNAs in cancer pathways. Cancer Cell 29: 452-463, 2016.

7. Zhan Y, Chen Z, Li Y, He A, He S, Gong Y, Li X and Zhou L: Long non-coding RNA DANCR promotes malignant phenotypes of bladder cancer cells by modulating the miR-149/MSI2 axis as a ceRNA. J Exp Clin Cancer Res 37: 273, 2018.

8. Chen DL, Lu YX, Zhang JX, Wei XL, Wang F, Zeng ZL, Pan ZZ, Yuan YF, Wang FH, Pelicano H, et al: Long non-coding RNA UICLM promotes colorectal cancer liver metastasis by acting as a ceRNA for microRNA-215 to regulate ZEB2 expression. Theranostics 7: 4836-4849, 2017.

9. Hong Q, Li O, Zheng W, Xiao WZ, Zhang L, Wu D, Cai GY, He JC and Chen XM: LncRNA HOTAIR regulates HIF-1 $\alpha / A X L$ signaling through inhibition of miR-217 in renal cell carcinoma. Cell Death Dis 8: e2772, 2017.

10. Zhou W, Ye XL, Xu J, Cao MG, Fang ZY, Li LY, Guan GH, Liu Q, Qian YH and Xie D: The lncRNA H19 mediates breast cancer cell plasticity during EMT and MET plasticity by differentially sponging miR-200b/c and let-7b. Sci Signal 10: eaak9557, 2017.

11. Trimarchi T, Bilal E, Ntziachristos P, Fabbri G, Dalla-Favera R, Tsirigos A and Aifantis I: Genome-wide mapping and characterization of Notch-regulated long noncoding RNAs in acute leukemia. Cell 158: 593-606, 2014

12. Atianand MK, Hu W, Satpathy AT, Shen Y, Ricci EP, Alvarez-Dominguez JR, Bhatta A, Schattgen SA, McGowan JD, Blin J, et al: A long noncoding RNA lincRNA-EPS acts as a transcriptional brake to restrain inflammation. Cell 165: 1672-1685, 2016.

13. Kopp F and Mendell JT: Functional classification and experimenta dissection of long noncoding RNAs. Cell 172: 393-407, 2018.

14. Tamang S, Acharya V, Roy D, Sharma R, Aryaa A, Sharma U, Khandelwal A, Prakash H, Vasquez KM and Jain A: SNHG12: An LncRNA as a potential therapeutic target and biomarker for human cancer. Front Oncol 9: 901, 2019.

15. Jin XJ, Chen XJ, Zhang ZF, Hu WS, Ou RY, Li S, Xue JS, Chen LL, $\mathrm{Hu} \mathrm{Y}$ and $\mathrm{Zhu} \mathrm{H}$ : Long noncoding RNA SNHG12 promotes the progression of cervical cancer via modulating miR-125b/STAT3 axis. J Cell Physiol 234: 6624-6632, 2019.

16. Dong J, Wang Q, Li L and Xiao-Jin Z: Upregulation of long non-coding RNA small nucleolar RNA host gene 12 contributes to cell growth and invasion in cervical cancer by acting as a sponge for MiR-424-5p. Cell Physiol Biochem 45: 2086-2094, 2018.

17. Jiang B, Hailong S, Yuan J, Zhao H, Xia W, Zha Z, Bin W and Liu Z: Identification of oncogenic long noncoding RNA SNHG12 and DUXAP8 in human bladder cancer through a comprehensive profiling analysis. Biomed Pharmacother 108: 500-507, 2018.

18. Zhou B, Li L, Li Y, Sun H and Zeng C: Long noncoding RNA SNHG12 mediates doxorubicin resistance of osteosarcoma via miR-320a/MCL1 axis. Biomed Pharmacother 106: 850-857, 2018.

19. Sun Y, Liu J, Chu L, Yang W, Liu H, Li C and Yang J: Long noncoding RNA SNHG12 facilitates the tumorigenesis of glioma through miR-101-3p/FOXP1 axis. Gene 676: 315-321, 2018.

20. Chen Q, Zhou W, Du SQ, Gong DX, Li J, Bi JB, Li ZH, Zhang Z, Li ZL, Liu XK and Kong CZ: Overexpression of SNHG12 regulates the viability and invasion of renal cell carcinoma cells through modulation of HIF1 $\alpha$. Cancer Cell Int 19: 128, 2019.

21. Wang O, Yang F, Liu Y, Lv L, Ma R, Chen C, Wang J, Tan Q, Cheng Y, Xia E, et al: C-MYC-induced upregulation of lncRNA SNHG12 regulates cell proliferation, apoptosis and migration in triple-negative breast cancer. Am J Transl Res 9: 533-545, 2017.

22. Liu ZB, Tang C, Jin X, Liu SH and Pi W: Increased expression of lncRNA SNHG12 predicts a poor prognosis of nasopharyngeal carcinoma and regulates cell proliferation and metastasis by modulating Notch signal pathway. Cancer Biomark 23: 603-613, 2018.

23. Xian HP, Zhuo ZL, Sun YJ, Liang B and Zhao XT: Circulating long non-coding RNAs HULC and ZNFX1-AS1 are potential biomarkers in patients with gastric cancer. Oncol Lett 16 : 4689-4698, 2018

24. Moch H, Cubilla AL, Humphrey PA, Reuter VE and Ulbright TM The 2016 WHO classification of tumours of the urinary system and male genital organs-part a: Renal, penile, and testicular tumours. Eur Urol 70: 93-105, 2016.

25. Ricketts CJ, De Cubas AA, Fan H, Smith CC, Lang M, Reznik E, Bowlby R, Gibb EA, Akbani R, Beroukhim R, et al: The cancer genome atlas comprehensive molecular characterization of renal cell carcinoma. Cell Rep 23: 313-326.e315, 2018.

26. Livak KJ and Schmittgen TD: Analysis of relative gene expression data using real-time quantitative PCR and the 2(-Delta Delta C(T)) method. Methods 25: 402-408, 2001.
27. Boguslawska J,Kedzierska H,Poplawski P, Rybicka B, Tanski Z and Piekielko-Witkowska A: Expression of genes involved in cellular adhesion and extracellular matrix remodeling correlates with poor survival of patients with renal cancer. J Urol 195: 1892-1902, 2016.

28. Bhan A, Soleimani M and Mandal SS: Long noncoding RNA and cancer: A new paradigm. Cancer Res 77: 3965-3981, 2017.

29. Fang Y and Fullwood MJ: Roles, functions, and mechanisms of long non-coding RNAs in cancer. Genomics Proteomics Bioinformatics 14: 42-54, 2016.

30. Yarani R, Mirza AH, Kaur S and Pociot F: The emerging role of lncRNAs in inflammatory bowel disease. Exp Mol Med 50: 1-14, 2018.

31. Hirata H, Hinoda Y, Shahryari V, Deng G, Nakajima K, Tabatabai ZL, Ishii $\mathrm{N}$ and Dahiya R: Long noncoding RNA MALAT1 promotes aggressive renal cell carcinoma through Ezh2 and interacts with miR-205. Cancer Res 75: 1322-1331, 2015.

32. Lan T, Ma W, Hong Z, Wu L, Chen X and Yuan Y: Long non-coding RNA small nucleolar RNA host gene 12 (SNHG12) promotes tumorigenesis and metastasis by targeting miR-199a/b-5p in hepatocellular carcinoma. J Exp Clin Cancer Res 36: 11, 2017.

33. Ding S, Qu W, Jiao Y, Zhang J, Zhang C and Dang S: LncRNA SNHG12 promotes the proliferation and metastasis of papillary thyroid carcinoma cells through regulating wnt/ $\beta$-catenin signaling pathway. Cancer Biomark 22: 217-226, 2018.

34. Wang P, Chen D, Ma H and Li Y: LncRNA SNHG12 contributes to multidrug resistance through activating the MAPK/Slug pathway by sponging miR-181a in non-small cell lung cancer. Oncotarget 8: 84086-84101, 2017.

35. Qi X, Zhang DH, Wu N, Xiao JH, Wang X and Ma W: ceRNA in cancer: Possible functions and clinical implications. J Med Genet 52: 710-718, 2015.

36. Maolakuerban N, Azhati B, Tusong H, Abula A, Yasheng A and Xireyazidan A: MiR-200c-3p inhibits cell migration and invasion of clear cell renal cell carcinoma via regulating SLC6A1. Cancer Biol Ther 19: 282-291, 2018.

37. Jiang J, Yi BO, Ding S, Sun J, Cao W and Liu M: Demethylation drug 5-Aza-2'-deoxycytidine-induced upregulation of miR-200c inhibits the migration, invasion and epithelial-mesenchymal transition of clear cell renal cell carcinoma in vitro. Oncol Lett 11: 3167-3172, 2016.

38. Wang X, Chen X, Han W, Ruan A, Chen L, Wang R, Xu Z, Xiao P, Lu X, Zhao Y, et al: miR-200c targets CDK2 and suppresses tumorigenesis in renal cell carcinoma. Mol Cancer Res 13: $1567-1577,2015$.

39. Gao C, Peng FH and Peng LK: MiR-200c sensitizes clear-cell renal cell carcinoma cells to sorafenib and imatinib by targeting heme oxygenase-1. Neoplasma 61: 680-689, 2014.

40. Ding J, Yeh CR, Sun Y, Lin C, Chou J, Ou Z, Chang C, Qi J and Yeh S: Estrogen receptor $\beta$ promotes renal cell carcinoma progression via regulating LncRNA HOTAIR-miR-138/200c/204/217 associated CeRNA network. Oncogene 37: 5037-5053, 2018.

41. Ying $\mathrm{G}, \mathrm{Wu} \mathrm{R}, \mathrm{Xia} \mathrm{M}, \mathrm{Fei}, \mathrm{X}, \mathrm{He} \mathrm{QE}$, Zha C and Wu F: Identification of eight key miRNAs associated with renal cell carcinoma: A meta-analysis. Oncol Lett 16: 5847-5855, 2018.

42. Wu YH, Chang TH, Huang YF, Chen CC and Chou CY: COL11A confers chemoresistance on ovarian cancer cells through the activation of $\mathrm{Akt} / \mathrm{c} / \mathrm{EBP} \beta$ pathway and PDK1 stabilization. Oncotarget 6: 23748-23763, 2015

43. Wu YH, Chang TH, Huang YF, Huang HD and Chou CY: COL11A1 promotes tumor progression and predicts poor clinical outcome in ovarian cancer. Oncogene 33: 3432-3440, 2014.

44. Jia D, Liu Z, Deng N, Tan TZ, Huang RY, Taylor-Harding B, Cheon DJ, Lawrenson K, Wiedemeyer WR, Walts AE, et al: A COL11A1-correlated pan-cancer gene signature of activated fibroblasts for the prioritization of therapeutic targets. Cancer Lett 382: 203-214, 2016.

45. Yang H, Wu J,Zhang J, Yang Z, Jin W, Li Y, Jin L, Yin L, Liu H and Wang Z: Integrated bioinformatics analysis of key genes involved in progress of colon cancer. Mol Genet Genomic Med 7: e00588, 2019.

46. Feldmann G, Habbe N, Dhara S, Bisht S, Alvarez H, Fendrich V, Beaty R, Mullendore M, Karikari C, Bardeesy N, et al: Hedgehog inhibition prolongs survival in a genetically engineered mouse model of pancreatic cancer. Gut 57: 1420-1430, 2008.

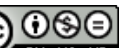

This work is licensed under a Creative Commons Attribution-NonCommercial-NoDerivatives 4.0 International (CC BY-NC-ND 4.0) License. 